\title{
Anatomical Reconstruction and Functional Imaging Reveal an Ordered Array of Skylight Polarization Detectors in Drosophila
}

\author{
๑Deter T. Weir, ${ }^{1 *}{ }^{\oplus}$ Miriam J. Henze, ${ }^{2 *}$ Christiane Bleul, ${ }^{2}$ Franziska Baumann-Klausener, ${ }^{2}$ Thomas Labhart, ${ }^{2}$ \\ and ${ }^{\circledR}$ Michael H. Dickinson ${ }^{1}$ \\ ${ }^{1}$ Division of Biology and Biological Engineering, California Institute of Technology, Pasadena, California 91125, and ${ }^{2}$ Institute of Molecular Life Sciences, \\ University of Zurich, 8057 Zurich, Switzerland
}

\begin{abstract}
Many insects exploit skylight polarization as a compass cue for orientation and navigation. In the fruit fly, Drosophila melanogaster, photoreceptors R7 and R8 in the dorsal rim area (DRA) of the compound eye are specialized to detect the electric vector (e-vector) of linearly polarized light. These photoreceptors are arranged in stacked pairs with identical fields of view and spectral sensitivities, but mutually orthogonal microvillar orientations. As in larger flies, we found that the microvillar orientation of the distal photoreceptor R7 changes in a fan-like fashion along the DRA. This anatomical arrangement suggests that the DRA constitutes a detector for skylight polarization, in which different e-vectors maximally excite different positions in the array. To test our hypothesis, we measured responses to polarized light of varying e-vector angles in the terminals of R7/8 cells using genetically encoded calcium indicators. Our data confirm a progression of preferred e-vector angles from anterior to posterior in the DRA, and a strict orthogonality between the e-vector preferences of paired R7/8 cells. We observed decreased activity in photoreceptors in response to flashes of light polarized orthogonally to their preferred e-vector angle, suggesting reciprocal inhibition between photoreceptors in the same medullar column, which may serve to increase polarization contrast. Together, our results indicate that the polarization-vision system relies on a spatial map of preferred e-vector angles at the earliest stage of sensory processing.
\end{abstract}

Key words: insect; navigation; polarization opponency; polarized light; vision

Significance Statement

The fly's visual system is an influential model system for studying neural computation, and much is known about its anatomy, physiology, and development. The circuits underlying motion processing have received the most attention, but researchers are increasingly investigating other functions, such as color perception and object recognition. In this work, we investigate the early neural processing of a somewhat exotic sense, called polarization vision. Because skylight is polarized in an orientation that is rigidly determined by the position of the sun, this cue provides compass information. Behavioral experiments have shown that many species use the polarization pattern in the sky to direct locomotion. Here we describe the input stage of the fly's polarizationvision system.

\section{Introduction}

Light falling on the retina provides abundant information about the environment. Patterns of light and dark indicate the shape and position of three-dimensional objects, while color provides

Received Jan. 27, 2016; revised April 4, 2016; accepted April 6, 2016.

Author contributions: P.T.W., M.J.H., T.L., and M.H.D. designed research; P.T.W., M.J.H., C.B., and F.B.-K. performed research; P.T.W., M.J.H., T.L., and M.H.D. analyzed data; P.T.W., M.J.H., T.L., and M.H.D. wrote the paper.

This work was supported by the National Institute of Neurological Disorders and Stroke of the National Institutes of Health under Award U01NS090514 (M.H.D.), the National Science Foundation under Grant No. 1352707 (M.H.D.), the Paul G. Allen Family Foundation (M.H.D.), and the German National Merit Foundation (Studienstiftung des deutschen Volkes; M.J.H.). The content is solely the responsibility of the authors and does not necessarily represent the official views of the National Institutes of Health. We thank S. Sommer and J. Smolka for advice on statistics, M. clues about the chemical and structural properties of materials. In addition to these familiar aspects of vision, many arthropods perceive the polarization of light (Horváth, 2014). When light is linearly polarized, its constituent electric fields are aligned along

Wernet for providing prh1-eGFP transgenic flies, B. Schnell for valuable discussions, and two anonymous reviewers for constructive comments.

The authors declare no competing financial interests.

*P.T.W. and M.J.H. contributed equally to this work.

Correspondence should be addressed to either Peter T. Weir at the above address or Miriam J. Henze at the above address. E-mail:weir@caltech.edu or miri@mhenze.net.

DOI:10.1523/JNEUROSCI.0310-16.2016

Copyright $\odot 2016$ the authors $\quad 0270-6474 / 16 / 365397-08 \$ 15.00 / 0$ 
one axis, called the electric-vector (e-vector), which lies in the plane perpendicular to the direction of propagation. Among other natural sources of polarized light, sunlight scattered by air molecules of the atmosphere (skylight) is polarized in a pattern relative to the position of the sun. This process renders celestial polarization a useful compass cue for insects during locomotion.

In many species of insects, a specialized portion of the compound eye, known as the dorsal rim area (DRA), mediates the detection of e-vector angles (Labhart and Meyer, 1999). The rhabdomeric photoreceptors of insects are inherently sensitive to linear polarization because the microvilli, which contain photopigments, extend at right angles to the optical axis. As a result of both the tubular shape of each microvillus and a directional anchorage of the pigment molecules in the membrane, photon absorption is maximal for light with an e-vector parallel to the microvillar axis (Moody and Parriss, 1961; Israelachvili and Wilson, 1976; Goldsmith and Wehner, 1977; Land, 1991; Roberts et al., 2011). A previous study has shown that the central photoreceptors R7 and R8 in the DRA of the fruit fly, Drosophila melanogaster, are specialized for the detection of polarized light (Wernet et al., 2012). These photoreceptors express the same visual pigment (Fortini and Rubin, 1990) and share an optical axis, because the rhabdomere of R8 lies directly below that of R7 (Wada, 1974; Wernet et al., 2012). Their untwisted rhabdomeres exhibit well aligned microvilli, which indicates high polarization sensitivity (Wernet et al., 2012). Identical adaptations have been observed in larger flies (Wunderer and Smola, 1982; Strausfeld and Wunderer, 1985).

In Musca and Calliphora, intracellular recordings have revealed that the central photoreceptors in the DRA respond to ultraviolet light, are highly sensitive to changes in e-vector angle, and exhibit e-vector preferences that correlate with their relative position (Hardie, 1984). Furthermore, the microvillar orientation of R7 and R8 are approximately orthogonal, both in larger flies and in Drosophila (Wada, 1974; Wunderer and Smola, 1982; Wernet et al., 2012). It is thought that this arrangement allows for the comparison of the signals of the two central cells by polarization-opponent neurons, although this possibility has not been confirmed by simultaneous recordings from both photoreceptors. The output of polarization-opponent neurons could provide a luminance-independent signal of increased polarization contrast for further processing, as observed in other insects (Wehner and Labhart, 2006; Heinze, 2014).

Although the ability of Drosophila to orient relative to the e-vector of polarized light is established (Stephens et al., 1953; Wolf et al., 1980; Weir and Dickinson, 2012; Wernet et al., 2012; Velez et al., 2014), little is known about the neuronal computations underlying the behavior in this genetic model organism. To approach this question, we investigated the input stage of the polarization-vision pathway. First, we examined the anatomy of the retina and the optics of the compound eye in the DRA. To study the responses of central photoreceptors to polarized light, we then observed the neural activity in their output terminals in the medulla, the second optic neuropil.

\section{Materials and Methods}

Optical axes of photoreceptors. We determined the optical axes of photoreceptors by observing corneal pseudopupils in both compound eyes of 11 males and 12 females of the wild-type strain Oregon-R under orthodromic illumination. Each fly was mounted in a goniometer such that there was no yaw deviation from the sagittal plane of the body, while pitch and roll could be adjusted independently. In Drosophila, rows of ommatidia originate at six fairly stable positions from the border of the

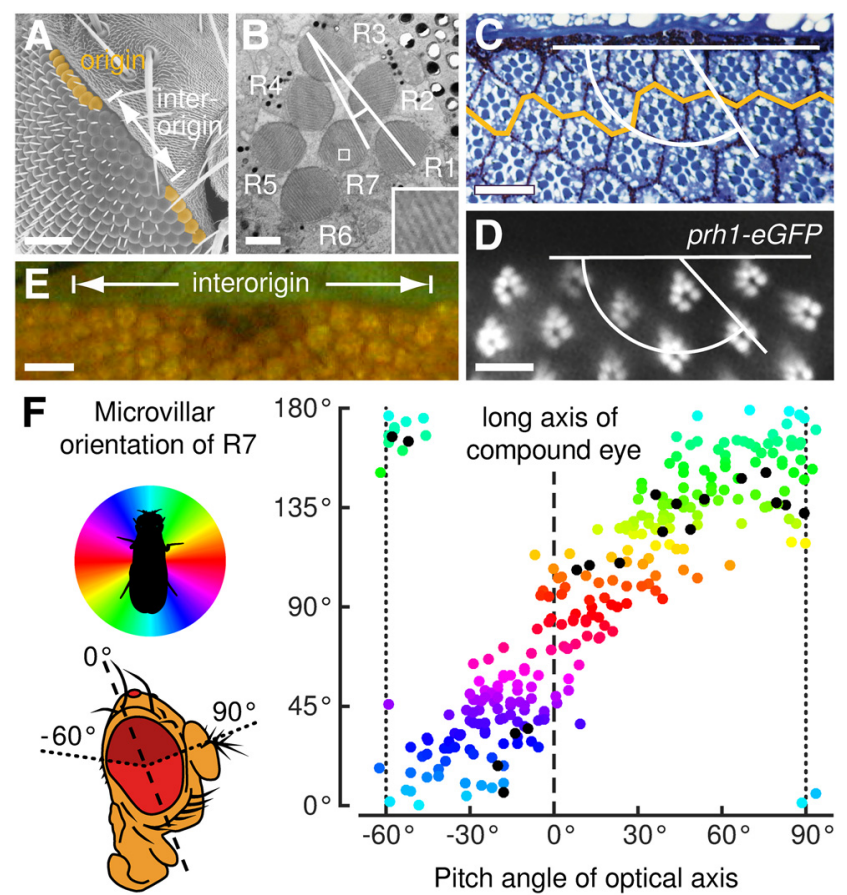

Figure 1. Prediction of e-vector preferences of R7 photoreceptors in the DRA of Drosophila. $A$, Scanning electron micrograph of the right compound eye highlighting landmarks used for measurements. $\boldsymbol{B}-\boldsymbol{D}$, Unlike in the rest of the eye, the rhabdomere of $\mathrm{R} 7$ is similar in diameter to the rhabdomeres of R1-R6 in the DRA ( $\boldsymbol{B} ; \boldsymbol{C}$, ommatidia above orange line). On electron microscopic sections, we determined the microvillar orientation of such $R 7$ cells relative to the axis through the rhabdomeres of R1-R3 $(\boldsymbol{B})$, which in turn was measured relative to the margin of the eye on light microscopic sections ( $\boldsymbol{C}$ ) and in flies expressing eGFP in R1-R6 under control of a minimal Rh1 promoter (D). $\boldsymbol{E}$, Furthermore, we determined the optical axes of photoreceptors in relation to the long axis of the compound eye (pitch) and the sagittal plane of the head (roll) by rotating the fly in a goniometer until the pseudopupil (dark spot) was centered on one of the landmarks. $\boldsymbol{F}$, Based on these measurements, we calculated the angle between the microvilli of R7 in the right eye and the sagittal plane, and plotted it against the position of the receptor given by the pitch angle of its optical axis. Anatomical results were collected from 28 flies and together covered the entire DRA. Data of a single eye are shown in black. Upper left, Color-coding of microvillar orientation. Lower left, Natural head posture of a fly standing on a horizontal surface (up, zenith). Scale bars: $\boldsymbol{A}, 50 \mu \mathrm{m} ; \boldsymbol{B}, 1 \mu \mathrm{m} ; \boldsymbol{C}, \boldsymbol{D}, 10 \mu \mathrm{m} ; \boldsymbol{E}, 25 \mu \mathrm{m}$. Image in $\boldsymbol{A}$ courtesy of E. Meyer.

compound eyes (Fig. 1A). We adopted these "origins" as landmarks, and rotated the fly until the pseudopupil was centered on an origin or between two neighboring origins, henceforth called "interorigins" (Fig. $1 E$ ). The angular deviations from the sagittal plane and from the long axis of the eye (axis of maximum diameter) were then read as roll and pitch, respectively. We found neither significant deviations from bilateral sym$\operatorname{metry}\left(F_{(1,36.9)}=0.16, p=0.69\right.$ for roll; $F_{(1,61)}=1.24, p=0.27$ for pitch $)$ nor significant differences between males and females $\left(F_{(1,20.6)}=0.37\right.$, $p=0.55$ for roll; $F_{(1,20.3)}=3.52, p=0.08$ for pitch; Mixed procedure in SAS 9.1.3, SAS Institute). Therefore we mirrored the measurements from left eyes, and combined the data for both eyes and across sexes.

Orientation of microvilli in $R 7$ photoreceptors. To determine the orientations of the microvilli of R7 photoreceptors in the DRA, heads of 28 wild-type flies of both sexes were fixed with $2 \%$ glutaraldehyde in $0.05 \mathrm{M}$ $\mathrm{Na}$-cacodylate buffer, $\mathrm{pH} 7.2-7.4$, at $4^{\circ} \mathrm{C}$ overnight, postfixed with $2 \%$ $\mathrm{OsO}_{4}$ in distilled water at room temperature for $2 \mathrm{~h}$, and dehydrated in 2.2-dimethoxypropane for $20 \mathrm{~min}$ and in pure acetone for $45 \mathrm{~min}$. We embedded the tissue in Epon and cut ultrathin $(80-90 \mathrm{~nm})$ and semithin $(1 \mu \mathrm{m})$ tangential sections of the DRA on an Ultracut microtome (Leica Microsystems) using a diamond knife. Ultrathin sections were treated with uranyl acetate and lead citrate, and photographed under a Philips transmission electron microscope (CM100, FEI) with a Gatan digital camera. Semithin sections were stained with methylene blue and viewed under bright-field illumination in a BX61 microscope (Olympus) 


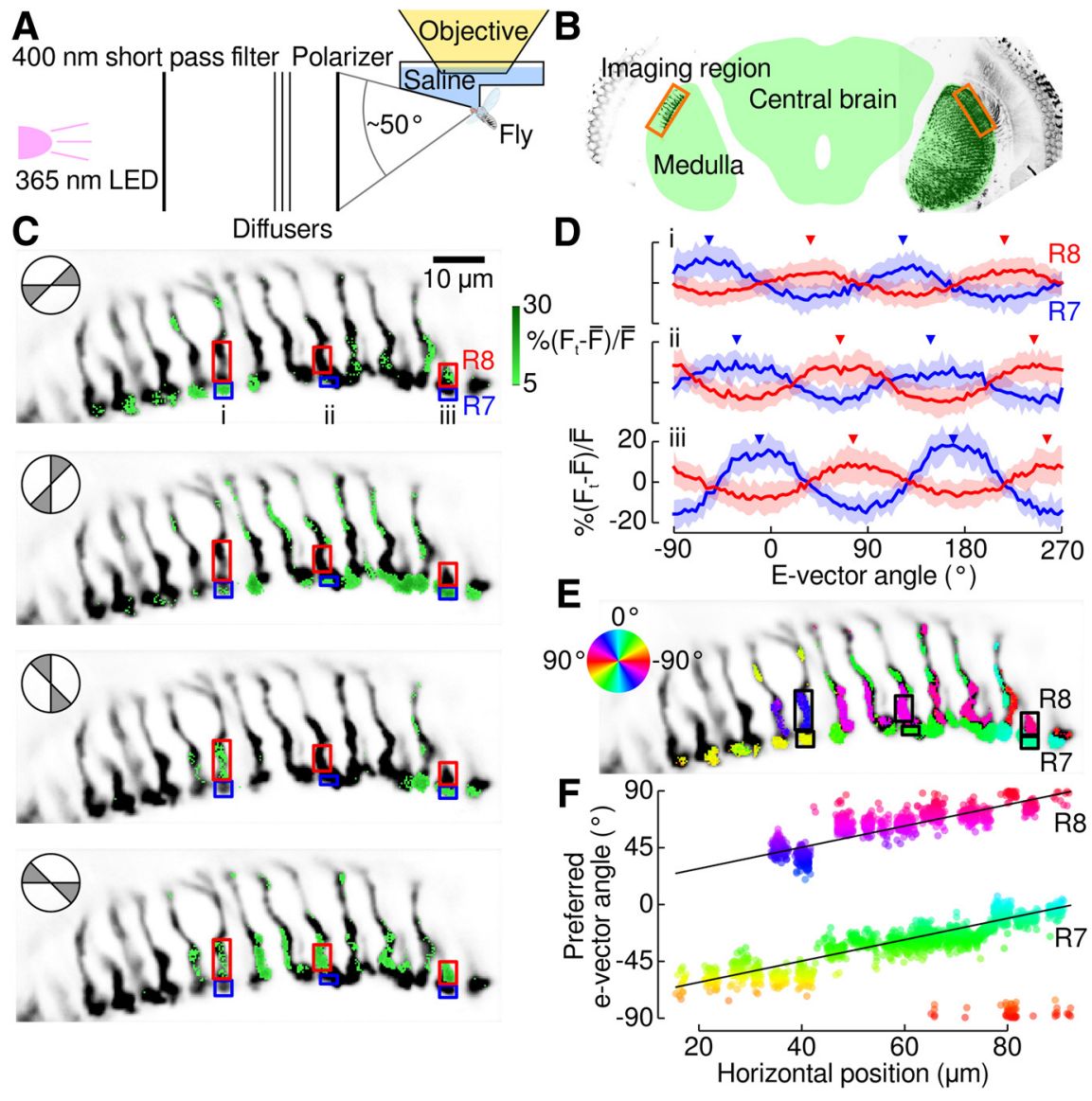

Figure 2. Example responses of dorsal rim photoreceptors to rotation of the e-vector angle. $\boldsymbol{A}$, Schematic representation of experimental apparatus. $\boldsymbol{B}$, Imaging location (rectangles) in the dorsal rim area of the medulla (MEDRA) relative to other brain regions. Right, A maximum projection of fluorescence in a fly with Rh3-GAL4 driving expression of tdTomato (grayscale). Left, A single optical section from the same fly at the depth used for functional imaging. $\boldsymbol{C}$, Mean imaging frames for four ranges of polarizer orientation, indicated by icon in upper left. Changes in GCaMP6 fluorescence (percentage change of the instantaneous fluorescence, $F_{\mathrm{t}}$ from the mean fluorescence, $\left.\bar{F}\right)>5 \%$ are indicated in green, tdTomato fluorescence in grayscale. $D$, Fluorescence traces for the regions of interest shown in C. Data for one full rotation of the polarizing filter are plotted (mean and quartiles of pixels in the region). $\boldsymbol{E}$, Pseudocolor image showing the orientation of the polarizer eliciting the maximum response (preferred e-vector angle) for each pixel that exhibited significant polarization-triggered activity; tdTomato fluorescence in grayscale. $\boldsymbol{F}$, Preferred e-vector angle for every responding pixel in $\boldsymbol{E}$, plotted against horizontal position in the imaging frame. The trend line for this fly is shown in black. Note that the trend line is only defined modulo $90^{\circ}$, but reproduced twice here to emphasize that it matches responses of both R7 and R8. Color scale is shared with $\boldsymbol{E}$.

equipped with a Colorview IIIu camera (Olympus). First, we measured the angle between the microvilli of R7 and a straight line through the rhabdomeres of R1-R3 in 272 DRA ommatidia on electron microscopic sections (Fig. 1B). Next, we identified the same ommatidia on light microscopic sections and determined the orientation of R1-R3 with respect to the eye rim (Fig. 1C). To control for distortions, we repeated this step in vivo in the dorsal part of the compound eye of prh1-eGFP transgenic flies (Pichaud and Desplan, 2001). We mounted 28 males and 29 females on the wax bottom of a Petri dish and visualized eGFP expression in the six outer photoreceptors of each ommatidium under a BX61 fluorescence microscope with an F-View II camera (Olympus), optically neutralizing the cornea by water immersion (Fig. 1D). A comparison between both methods by fitting linear models to the data in R (R Foundation for Statistical Computing) did not yield differences that are likely to be of functional relevance (maximal difference is $16^{\circ}$, while values for each method scatter by $>60^{\circ}$ at a given position).

Prediction of e-vector preference of R7. By combining our optical and anatomical measurements in Matlab R2015a (MathWorks), it was possible to compute the e-vector that should maximally stimulate each R7 cell, i.e., to predict the e-vector preference of each cell. We first determined the optical axes of individual photoreceptors by dividing the change in pitch from one measuring point (origin or interorigin) to the next by the number of ommatidia in between, taking the spacing factors of the hexagonal array of ommatidia into account. The resulting interommatidial angles allowed us to calculate the pitch of any R7 cell based on the position of the ommatidium. We inferred the roll from the measurements at origins and interorigins by cubic spline interpolation, and computed the angle between the eye rim and the sagittal plane of the body for each position. Combining this angle with the angle between the eye rim and the microvilli of R7 yielded the predicted e-vector preference of R7, defined as the angle between its microvillar orientation and the sagittal plane. Finally, we plotted the predicted e-vector preference against the position of the receptor, which is given by the pitch deviation of its optical axis from the long axis of the compound eye (Fig. $1 F)$.

Functional imaging of photoreceptor terminals. As previously described (Maimon et al., 2010), we glued to a custom holder female flies $<10$ days posteclosion expressing $\geq 1$ copy of Rh3-GAL4, UAS-tdTomato (Shaner et al., 2004), and either UAS-GCaMP6f or UAS-GCaMP6s (Chen et al., 2013). All flies were the progeny of crosses between Bloomington stock numbers 4757, $36328,42747,42746$, or 42749 , and CantonS. We painted the holder white to minimize the difference in reflected intensities of different e-vector angles of polarized light. Immediately before each experiment, we dissected a hole in the cuticle directly posterior to the medulla on one side of the brain in standard saline or in saline with $3 \mathrm{~mm}$ calcium (Wilson et al., 2004). We imaged the part of the medulla that receives input from the DRA (Fig. $2 B$ ), which has been termed the DRA of the medulla (MEDRA) by previous authors (Ito et al., 2014). All data are reported for the right MEDRA, and we mirrored data from the left MEDRA. We used a Prairie Ultima IV 2-photon microscope equipped with a Nikon $40 \times$ NIR Apo objective lens to image $0.33 \times 0.33 \mu \mathrm{m}^{2}$ pixels each for 4 $\mu$ s with $930 \mathrm{~nm}$ laser light delivered by a Ti: Saphire laser (Coherent). The power at the back aperture of the objective lens never exceeded $26 \mathrm{~mW}$. Two multialkali photomultiplier tubes monitored emitted photons through either an HQ 525/50m-2p or an HQ 607/45-2p emission filter (Chroma Technology).

We exposed each fly to linearly polarized ultraviolet light $\left(\sim 10^{12}\right.$ photons $\mathrm{cm}^{-2} \mathrm{~s}^{-1}$, peak wavelength $378 \mathrm{~nm}, 10 \mathrm{~nm}$ full-width at halfmaximum) by directing the output of a light-emitting diode through a $400 \mathrm{~nm}$ short-pass filter, three fused silica ground glass diffusers (Edmund Optics), and a specialized ultraviolet-transmitting polarizing filter (Boulder Vision Optik), whose orientation was controlled via a stepper motor. This stimulus subtended an angle of $\sim 50^{\circ}$ (Fig. $2 A$ ).

In our first experiment, each trial consisted of a period of $8 \mathrm{~s}$ with the polarizer stationary, followed by five $360^{\circ}$ rotations of the polarizer at $22.5^{\circ} \mathrm{s}^{-1}$. We presented 18 trials to each fly, alternating between clockwise and counterclockwise rotations. In a subsequent experiment, we held the polarizer motionless in one or two positions and flashed light with a stationary e-vector angle on for $4 \mathrm{~s}$ and off for $4 \mathrm{~s}$ (a square wave intensity profile with $8 \mathrm{~s}$ period) 20 times. Three to five of these trials were presented to each fly.

Imaging analysis. To reduce the effects of brain motion, we adjusted the position of each image to maximize the spatial cross-correlation of 
the tdTomato signal in the image with a $512 \times 512$-pixel reference image acquired before each experiment. To detect changes in GCaMP6 fluorescence triggered by the rotation of the polarizer, we computed the Fourier transform of the time course of each pixel in the GCaMP channel for each trial during which the polarizer rotated clockwise. To eliminate the effects of delays and dynamics of the calcium indicator, we reversed the time course of each pixel for the trials in which the polarizer rotated counterclockwise, and then computed their Fourier transforms. Because the activity of an e-vector-sensitive photoreceptor should exhibit two peaks during each rotation of the polarizer, we determined the value of the averaged Fourier transforms of all trials at twice the rotation frequency $(0.125 \mathrm{~Hz})$. To make comparisons among different preparations easier, we normalized these values to the laser power, and computed their magnitude and phase. Dividing the phase by two yielded the e-vector angle that elicited the maximum response for each pixel, which we term the "preferred e-vector angle." The magnitude provided an indication of the strength of the modulation elicited by the rotation of the polarizer. To define a threshold for the strength of modulation above statistical noise, we randomly shuffled the Fourier transforms at $0.125 \mathrm{~Hz}$ of all trials of all flies, and then computed the distribution of norms for means of collections of 18 shuffled trials. We found the 99th percentile of the resulting distribution, and repeated this process 100 times. We then used the mean of all the 99th percentiles as our threshold for a "responding pixel."

To obtain an accurate estimate of the change of e-vector preference over the length of the DRA, we needed to sample simultaneously from a large number of columns across the MEDRA. Therefore, we only used data from flies in which $\geq 5 \%$ of all pixels surpassed our threshold for a significant response. Nine of 40 flies fulfilled this criterion. For each of these flies, we performed a linear regression of e-vector preference of each responding pixel versus its position along the MEDRA, numerically minimizing the sum squared angular difference between the trend line and four times the pixel's preferred e-vector angle (Weir, 2013). By multiplying the preferred e-vector angle by four, the orthogonal responses of each R7/8 pair are mapped to the same angle. Thus, the trend line minimized the error for all responding pixels regardless of cell type. To estimate the pitch of a receptor's optical axis, we scaled the position along the MEDRA by the ratio of the average interommatidial angle $\left[5.1^{\circ}\right.$; according to calculations by Stavenga (2003) for the whole eye, which agrees well with $5.06^{\circ}$, the value we measured for the dorsofrontal region of the DRA] to the average spacing between medullar columns (5.7 $\mu \mathrm{m}$ in our dataset). We then centered the resulting values on the known angular position of our stimulus.

To investigate changes in photoreceptor activity relative to their baseline activity in darkness, we presented flashes of light with a stationary e-vector angle to 36 flies, after completing the trials with the rotating polarizer. To identify pixels in the GCaMP6 images whose intensity decreased in response to the flashes, we performed a Fourier-based analysis similar to the one described above. For all pixels that had responded significantly to the rotating polarizer, we calculated the mean Fourier transform at 0.125 (one-eighth) $\mathrm{Hz}$ for the time courses in the flash experiment. Pixels that showed decreased GCaMP6 fluorescence to the light-on stimulus were identified by their negative phase in the Fourier transform.

\section{Results}

The DRA of Drosophila is a narrow strip along the dorsal eye rim and only 1-2 ommatidia wide (Tomlinson, 2003; Wernet et al., 2003). We determined the microvillar orientation of R7 photoreceptors in the entire DRA (Fig. $1 A-D$ ), which comprised on average 39 ommatidia in one compound eye (value derived from 55 light microscopic sections covering every region of the DRA $4-15$ times). This is considerably more than the $\sim 25$ ommatidia counted in the DRA of Drosophila in another study (Fortini and Rubin, 1991). A reason for this discrepancy might be that only a single row of DRA ommatidia was identified by the previous authors based on their staining technique, whereas we consistently detected up to two rows in the more dorsal parts of the DRA.
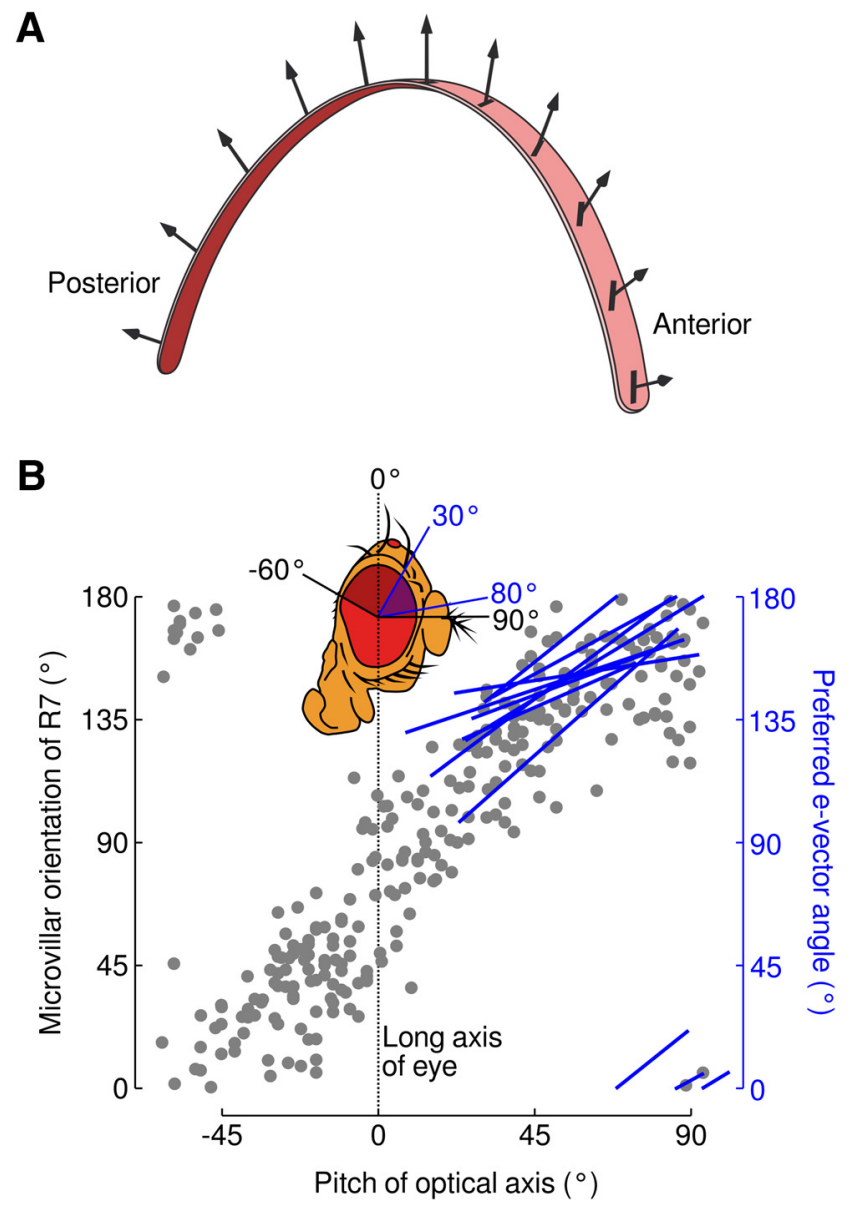

Figure 3. A, The optical axes of DRA photoreceptors in Drosophila are directed in a narrow arc close to the sagittal plane of the body at vastly different parts of the sky (arrows), including the zenith and areas near the horizon. Bars indicate the predicted e-vector preferences of R7 cells in the right eye projected on the upper visual hemisphere. $\boldsymbol{B}$, The array of $\mathrm{R} 7$ photoreceptors in the DRA of each eye systematically samples the entire range of possible e-vector angles. The data of our anatomical study (gray dots; Fig. $1 F$ ) are in close agreement with the trend lines from the functional imaging experiments (blue lines; Fig. 2F, black trend line). Two ordinate axes (left and right) are used to emphasize that the results originate from independent experiments.

In bigger flies (Wunderer and Smola, 1982; Strausfeld and Wunderer, 1985) and many other insects (Horváth, 2014), the microvillar orientation has been described to change in a fan-like manner along the DRA. We observed considerable deviations from such an arrangement in Drosophila (Fig. $1 F$ ), resulting in a scatter of up to $80^{\circ}$ (SD of residuals: $18^{\circ}$ ). However, on average the microvillar orientation of R7 changed continuously along the DRA, being approximately parallel to the sagittal plane of the body at the posterior end of the DRA and progressing counterclockwise (in the right eye) through slightly more than $180^{\circ}$, until lying again approximately parallel to the sagittal plane at the anterior end of the DRA. The optical axes of all DRA ommatidia were directed close to the sagittal plane. From the posterior to the anterior end of the DRA, they covered a wide range of $>150^{\circ}$ (Fig. $1 F$, inset), sampling the sky along a narrow arc that extended almost across the entire upper hemisphere (Fig. $3 A$ ).

To study the physiological properties of the central photoreceptors, we imaged calcium activity in R7/8 cell terminals in the medulla. In the DRA, both central photoreceptors express the ultraviolet-sensitive rhodopsin Rh3 (Fortini and Rubin, 1990). We used the Rh3-GAL4 driver line to express UAS-GCaMP6 and 
UAS-tdTomato in these photoreceptors, and imaged both fluorophores using two-photon microscopy (Fig. 2A,B).

The terminals of R7 cells project to a slightly more proximal layer in the medulla than those of R8 cells (Fischbach and Dittrich, 1989). However, because our driver line targeted both photoreceptor classes, we could not always unambiguously distinguish the terminals originating from R7 and R8 cells in the same column. In some cases, the cells were discernable by differences in stimulus-elicited changes of GCaMP6 fluorescence (Fig. $2 C, D)$. We identified the e-vector angle that elicited the largest response in each pixel, which we termed the preferred e-vector angle, and used the magnitude of the stimulus-elicited modulation to determine which pixels were significantly responsive (Fig. $2 E$ ). Based on our anatomical finding that $\mathrm{R} 7$ cells in this region have microvilli oriented at $\sim 135^{\circ}$ to the sagittal plane, we reasoned that they are more likely to have preferred e-vector angles at this value. In all flies in which $\geq 1 \%$ of all pixels were significantly responsive (18 of 40 flies), we observed groups of pixels with preferred e-vector angles near this value, which allowed us to classify them as belonging to R7. As expected, these cells invariably projected deeper into the medulla and terminated in a characteristic club shape, although they were intertwined with pixels of orthogonal e-vector preferences in the more distal parts of the medulla. It is likely that pixels with orthogonal tuning to those of the $\mathrm{R} 7$ cells represent R8 cells. After detrending the data for flies with many responding columns (see below), we used the $k$-means algorithm (Pedregosa et al., 2011) to cluster the e-vector preference angles on the unit circle of the significantly responding pixels into two groups for every fly. These clusters were separated by $86.4 \pm 7.5^{\circ}$ (mean $\pm \mathrm{SD} ; N=18$ flies), indicating that the preferred e-vectors of R7 and R8 cells are consistently orthogonal.

We also observed a systematic shift in the preferred e-vector angles across different columns of the medulla (Fig. $2 F$ ). R7 cells in ommatidia that receive light from more anterior directions terminate in the more ventrolateral part of the medulla due to the optic chiasma between the lamina and the medulla (Fortini and Rubin, 1991). These photoreceptors were most strongly responsive to e-vectors approximately parallel to the sagittal plane of the head, whereas those that view more dorsal parts of space and terminate in the more dorsomedial medulla prefer e-vectors normal to the sagittal plane. This pattern of e-vector preferences matches the systematic rotation of microvillar orientations that we observed anatomically (Fig. 3B), and suggests a retinotopic projection of the DRA photoreceptors to the medulla, which has also been described in locusts (Schmeling et al., 2015).

Closer examination of the raw data in Figure $2 \mathrm{~F}$ indicates that, although the preferred e-vector angles progress regularly according to the fan-like arrangement of the microvilli, individual columns often show preferences for e-vector angles that deviate from the trend line. We observed a similar phenomenon in the anatomical data of individual flies (Fig. $1 F$ ). This noise in the preferred e-vector angles along the DRA might affect the cell types independently. Alternatively, processes during development might act to maintain a tight orthogonality between the tuning of R7/8 cells in the same column. To assess these hypotheses, we compared the preferred e-vector angles for pixels with the same horizontal position in our imaging field of view for flies with many responding columns (flies with $\geq 5 \%$ of pixels responding significantly). In Figure 4, we plot the probability density function of absolute differences $>45^{\circ}$ between preferred e-vector angles of pairs of responding pixels with the same horizontal position. The peak at $90^{\circ}$ indicates that the preferred e-vector of R7 is in most cases orthogonal to that of the R8 cell in

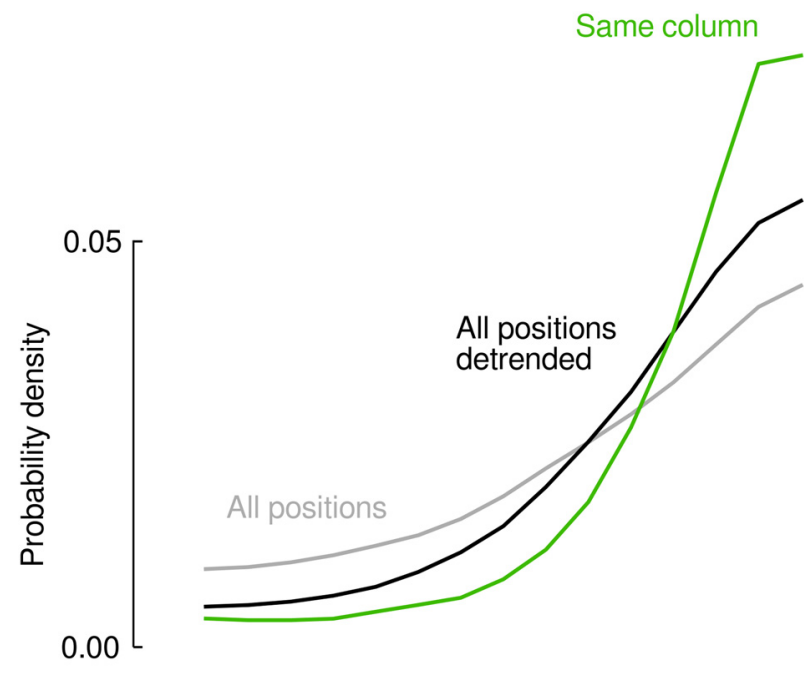

45

90

Figure 4. Photoreceptors in the same medullar column respond to orthogonal e-vectors. The probability density function (using $3^{\circ}$-wide bins) for the absolute value of the difference between every pair of responding pixels with the same horizontal position is shown in green (Fig. $2 F$, colored points with the same $x$-position). To exclude pixels with the same e-vector preference, only differences $>45^{\circ}$ are plotted. The peak indicates that the most common difference between e-vector preferences is $90^{\circ}$. The distributions are less tightly peaked for pairs of responding pixels independent of position in the MEDRA (gray) or after detrending the data by subtracting the value of the trend line (black), which shows that the linkage of e-vector preferences between $\mathrm{R} 7$ and $\mathrm{R} 8$ in the same ommatidium is more consistent than that between neighboring ommatidia.

the same column. To compare this orthogonality to the variability among columns, we subtracted the trend line of the shift in preferred e-vector angle across horizontal positions for each fly (Fig. 2F, black line). We then computed the absolute angular difference between all pairs of responding pixels in this detrended data. Because the distribution of pairwise differences in the detrended responses are not as tightly peaked around $90^{\circ}$, we conclude that the preferred e-vector angles of R7 and R8 within a column are more tightly linked to each other than they are to cells in the neighboring columns.

Although the alignment of microvilli endows a photoreceptor with intrinsic polarization sensitivity, it is possible that extrinsic signaling further sharpens its tuning. To search for such interactions, we analyzed the responses to flashes of light polarized at a constant e-vector angle, after evaluating the e-vector preference and photoreceptor type for each pixel as described above. Preferential absorption along the microvillar axis causes a photoreceptor to respond maximally to light polarized along that axis, but even light polarized orthogonally to the preferred e-vector angle is absorbed with lower probability. Thus, some increase in activity is expected in response to a light flash with any e-vector angle. In contrast to this expectation, we found that some R7 photoreceptors exhibited a decrease in the GCaMP signal in response to a light-on stimulus (Fig. 5A). This decrease suggests an external source of inhibition that was sufficiently large to overcome the direct excitation caused by the light flash. The most parsimonious explanation for this inhibition is a negative interaction with the $\mathrm{R} 8$ cell in the same medullar column, which, as expected, showed increased activity to the same light flash (Fig. 5A). In Figure 5C, we plot the proportion of pixels whose intensity decreased as a 

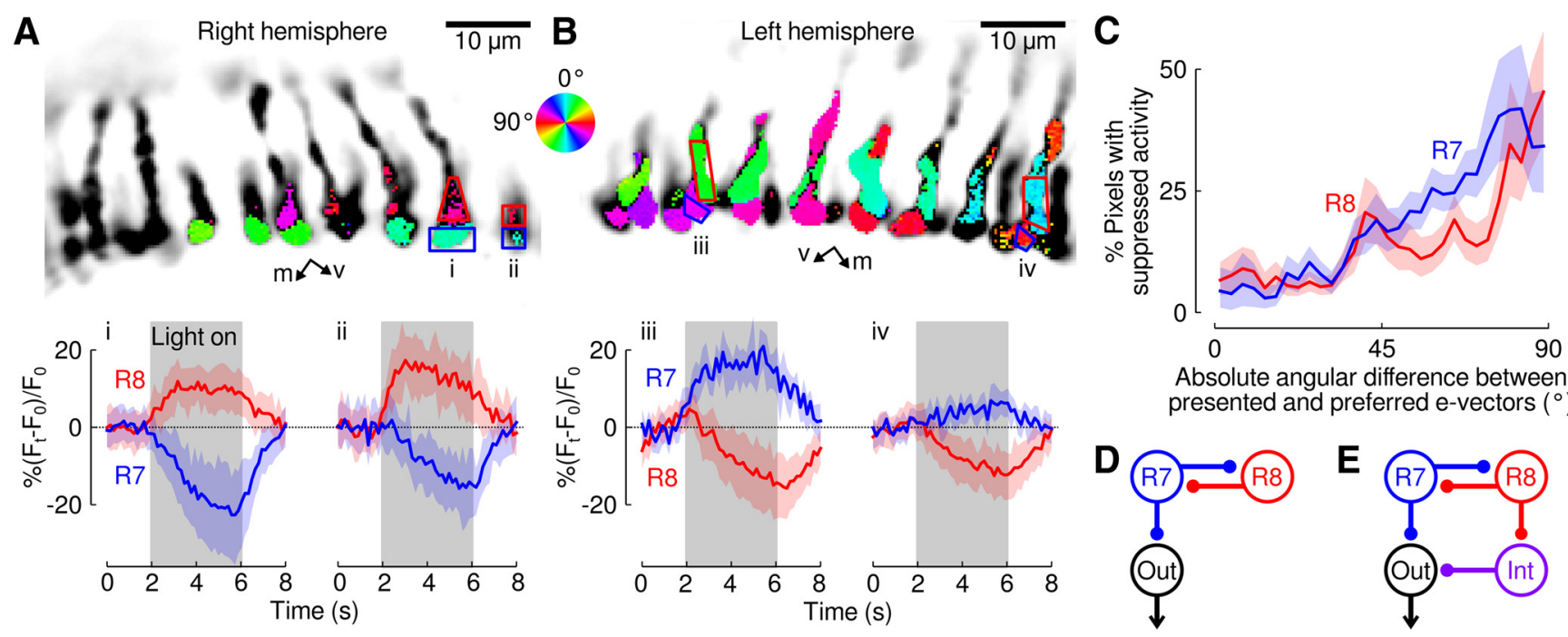

Figure 5. The activity of some photoreceptors decreased in response to flashes of polarized light. $\boldsymbol{A}, \boldsymbol{B}$, top, Example pseudocolor image of preferred e-vector angles in the right $(\boldsymbol{A})$ and left ( $\boldsymbol{B})$ MEDRA of two flies. Medial ( $\mathrm{m}$ ) and ventral (v) directions indicated by arrows. $\boldsymbol{A}, \boldsymbol{B}$, bottom, Time courses (mean and quartiles) of GCaMP6 fluorescence (percentage change of the instantaneous fluorescence, $F_{t}$, from the mean fluorescence in the $2 \mathrm{~s}$ before the light-on stimulus, $F_{0}$ ) from regions depicted at top in response to a flash of light polarized normal to the sagittal plane; note suppression of activity in two R7 cells $(\boldsymbol{A})$ and two R8 cells $(\boldsymbol{B})$. C, Percentage of responding pixels from R8 cells (red) and R7 cells (blue) with different preferred e-vectors whose GCaMP6 fluorescence was reduced by flashes of polarized light ( $3^{\circ}$-wide bins, with $99 \%$ confidence intervals, $N=17$ flies). A cell is more likely to show decreased activity the closer the absolute angle between its preferred e-vector and the e-vector of the stimulus light is to $90^{\circ}$. D, E, Two schematic diagrams of polarization processing in a medullar column containing two photoreceptors, an output neuron (Out), and a sign-inverting interneuron (Int). All connections between cells are inhibitory. Although we present versions of the models in which R7 is directly connected to the output neuron, the identities of R7 and R8 could be exchanged in both models.

function of the difference between each pixel's preferred e-vector angle and the e-vector angle of the stimulus light. The fraction of pixels indicating suppressed activity was greatest when the e-vector of the stimulus light was at $90^{\circ}$ to the preferred e-vector of R7, which is precisely the condition that should maximally excite its paired R8 cell. Because of the high density of photoreceptor terminals in our imaging plane, we did not have confidence in our ability to segment individual cells of the same type, and thus performed our analysis on the basis of pixels. Although the number of pixels representing each terminal undoubtedly varied, we interpret the results based on the percentages of pixels to be approximately representative of the responses of the photoreceptor terminals themselves. It is important to note that we could only directly observe the influence of inhibition if it caused a decrease in the GCaMP signal below baseline, and not if it merely reduced the excitation in response to the light flash. For this reason, we suspect that the results in Figure $5 C$ underestimate the fraction of photoreceptors inhibited by their partners; instead, it is more likely that all R7 cells receive inhibition. We also found evidence for the reciprocal interaction; the activity of R8 cells decreased in response to light flashes (Fig. 5B), and the effect depended on the e-vector angle of the stimulus (Fig. 5C). Hardie (1984), who observed a similar phenomenon in Musca, suggested that this reciprocal inhibition might arise from nonsynaptic (i.e., "ephaptic") coupling between the two photoreceptors. However, recent reconstructions of circuitry based on serialsection electron microscopy of the medulla document the existence of reciprocal chemical synapses between R7 and R8 (Takemura et al., 2013). Even though these results were obtained outside the MEDRA, they support the possibility that inhibitory synaptic connections could exist between the terminals of DRA photoreceptors.

\section{Discussion}

We provide two independent lines of evidence that the preferred e-vector angle of the central photoreceptors changes systemati- cally along the length of the DRA. The orientation of the microvilli of the distal photoreceptor R7 matches the pattern of e-vector preferences derived from functional imaging, and both confirm that the fan-like arrangement of preferred e-vector angles described in other insects is conserved in Drosophila. Our methods allowed us to directly observe an ordered linear representation of e-vector preferences across the medulla of individual flies. Using functional imaging, we demonstrated that the physiological e-vector preferences of paired photoreceptors in a single medullar column are strictly orthogonal, confirming the predictions of a prior anatomical study (Wernet et al., 2012). We also observed evidence for reciprocal inhibitory connections between the photoreceptors in the same column.

Schematic diagrams for two models of polarization processing in each column of the MEDRA are presented in Figure 5D,E. In both models, reciprocal inhibition between one R7 and one R8 cell with orthogonal e-vector preferences enhances polarization contrast. If the gain of the reciprocal inhibition were adequately tuned, the output of either photoreceptor would provide a signal that was independent of luminance (Fig. 5D; output only indicated for R7). Such a model requires precisely tuned connections such that the excitation of either photoreceptor caused by an unpolarized light source would be exactly canceled by inhibition from its partner. Alternatively, a luminance-independent output could be achieved by computing the difference signal between the two photoreceptors in a postsynaptic neuron. Given that both R7 and R8 are thought to release the neurotransmitter histamine (Stuart, 1999), it is unlikely that they make direct antagonistic connections on the same postsynaptic neuron. Instead, a signinverting interneuron interposed between one of the photoreceptors and the downstream target would be required (Fig. 5E). Given the relevant spatial scale, the synaptic delay introduced by such an interneuron is unlikely to introduce computational errors within the required bandwidth of the system. In this configuration, each $\mathrm{R} 7 / 8$ receptor pair in combination with an 
interneuron and a polarization-opponent output neuron would act as a differential polarization sensor. Polarization-opponent neurons have been recorded in the visual systems of several insect species (for review, see Heinze, 2014).

Outside of the DRA, the R7/8 photoreceptors are used for color discrimination, which requires similar computations as polarization detection. In the retinae of some vertebrates, color opponency is sharpened by negative feedback from horizontal cells onto cone photoreceptors (Fuortes et al., 1973; Stell et al., 1975; Murakami et al., 1982), yet there is no consensus on whether the source of this negative feedback is synaptic or ephaptic in nature (for review, see Kamermans and Spekreijse, 1999; Kramer and Davenport, 2015). Regardless of the mechanism, negative input from coupled photoreceptors appears to be a circuit motif common to both vertebrates and insects that sharpens the tuning of individual photoreceptors.

To analyze the local e-vector orientation of any part of a visual scene, an animal must compare the output of $\geq 3$ polarization sensors with different e-vector preferences (Kirschfeld, 1972). Without using memory, this task requires that the detectors view the scene element simultaneously. Our anatomical reconstruction shows that the Drosophila DRA contains sensors with a wide range of e-vector tuning angles. However, due to both the curved structure of the DRA and the small photoreceptor acceptance angles (Henze, 2009), the polarization sensors of the DRA view vastly diverging parts of the celestial polarization pattern, ranging from the frontal to the caudal horizon via the zenith (Fig. $3 A$ ). Apparently, the Drosophila DRA is not designed to evaluate local e-vector orientations but exploits the combined polarization signals from different parts of the celestial hemisphere. Further experiments will be required to understand how downstream circuits implement the necessary algorithms to represent polarization information in a manner useful for guiding behavior.

\section{Notes}

Supplemental material for this article is available at http://ptweir.github. io/flyHolder/. Instructions for fabricating custom stages that permit functional imaging and electrophysiology in intact, behaving flies can be found in the online supplementary material. This material has not been peer reviewed.

\section{References}

Chen TW, Wardill TJ, Sun Y, Pulver SR, Renninger SL, Baohan A, Schreiter ER, Kerr RA, Orger MB, Jayaraman V, Looger LL, Svoboda K, Kim DS (2013) Ultrasensitive fluorescent proteins for imaging neuronal activity. Nature 499:295-300. CrossRef Medline

Fischbach K, Dittrich A (1989) The optic lobe of Drosophila melanogaster. I. A Golgi analysis of wild-type structure. Cell Tissue Res 258:441-475.

Fortini ME, Rubin GM (1990) Analysis of cis-acting requirements of the $\mathrm{Rh} 3$ and Rh4 genes reveals a bipartite organization to rhodopsin promoters in Drosophila melanogaster. Genes Dev 4:444-463. CrossRef Medline

Fortini ME, Rubin GM (1991) The optic lobe projection pattern of polarization-sensitive photoreceptor cells in Drosophila melanogaster. Cell Tissue Res 265:185-191. CrossRef Medline

Fuortes MG, Schwartz EA, Simon EJ (1973) Colour-dependence of cone responses in the turtle retina. J Physiol 234:199-216. CrossRef Medline

Goldsmith TH, Wehner R (1977) Restrictions on rotational and translational diffusion of pigment in the membranes of a rhabdomeric photoreceptor. J Gen Physiol 70:453-490. CrossRef Medline

Hardie RC (1984) Properties of photoreceptors R7 and R8 in dorsal marginal ommatidia in the compound eyes of Musca and Calliphora. J Comp Physiol A 154:157-165. CrossRef

Heinze S (2014) Polarized-light processing in insect brains: recent insights from the desert locust, the monarch butterfly, the cricket, and the fruit fly. In: Polarized light and polarization vision in animal sciences (Gábor Horváth, ed), pp 61-111. Berlin: Springer.
Henze M (2009) Two facets of insect vision: polarization sensitivity and visual pigments. Doctoral dissertation, University of Zürich.

Horváth G (2014) Polarized light and polarization vision in animal sciences. Berlin: Springer.

Israelachvili JN, Wilson M (1976) Absorption characteristics of oriented photopigments in microvilli. Biol Cybern 21:9-15. CrossRef Medline

Ito K, Shinomiya K, Ito M, Armstrong JD, Boyan G, Hartenstein V, Harzsch S, Heisenberg M, Homberg U, Jenett A, Keshishian H, Restifo LL, Rössler W, Simpson JH, Strausfeld NJ, Strauss R, Vosshall LB, Vosshall LB (2014) A systematic nomenclature for the insect brain. Neuron 81: 755-765. CrossRef Medline

Kamermans M, Spekreijse H (1999) The feedback pathway from horizontal cells to cones. Vision Res 39:2449-2468. Medline

Kirschfeld K (1972) Die notwendige Anzahl von Rezeptoren zur Bestimmung der Richtung des elektrischen Vektors linear polarisierten Lichtes. Z Naturforsch 27c:578-579.

Kramer RH, Davenport CM (2015) Lateral inhibition in the vertebrate retina: the case of the missing neurotransmitter. PLoS Biol 13: e1002322. CrossRef Medline

Labhart T, Meyer EP (1999) Detectors for polarized skylight in insects: a survey of ommatidial specializations in the dorsal rim area of the compound eye. Microsc Res Tech 47:368-379. Medline

Land MF (1991) Polarizing the world of fish. Nature 353:118-119. CrossRef

Maimon G, Straw AD, Dickinson MH (2010) Active flight increases the gain of visual motion processing in Drosophila. Nat Neurosci 13:393-399. CrossRef Medline

Moody MF, Parriss JR (1961) The discrimination of polarized light by Octopus: a behavioural and morphological study. Z Vgl Physiol 44:268-291.

Murakami M, Shimoda Y, Nakatani K, Miyachi E, Watanabe S (1982) GABA-mediated negative feedback and color opponency in carp retina. Jpn J Physiol 32:927-935. Medline

Pedregosa F, Varoquaux G, Gramfort A, Michel V, Thirion B, Grisel O, Blondel M, Prettenhofer P, Weiss R, Dubourg V (2011) Scikit-learn: machine learning in Python. J Mach Learn Res 12:2825-2830.

Pichaud F, Desplan C (2001) A new visualization approach for identifying mutations that affect differentiation and organization of the Drosophila ommatidia. Development 128:815-826. Medline

Roberts NW, Porter ML, Cronin TW (2011) The molecular basis of mechanisms underlying polarization vision. Philos Trans R Soc Lond B Biol Sci 366:627-637. CrossRef Medline

Schmeling F, Tegtmeier J, Kinoshita M, Homberg U (2015) Photoreceptor projections and receptive fields in the dorsal rim area and main retina of the locust eye. J Comp Physiol A Neuroethol Sens Neural Behav Physiol 201:427-440. CrossRef Medline

Shaner NC, Campbell RE, Steinbach PA, Giepmans BN, Palmer AE, Tsien RY (2004) Improved monomeric red, orange and yellow fluorescent proteins derived from Discosoma sp. red fluorescent protein. Nat Biotechnol 22:1567-1572. Medline

Stavenga DG (2003) Angular and spectral sensitivity of fly photoreceptors. II. Dependence on facet lens F-number and rhabdomere type in Drosophila. J Comp Physiol A Neuroethol Sens Neural Behav Physiol 189:189-202. Medline

Stell WK, Lightfood DO, Wheeler TG, Leeper HF (1975) Goldfish retina: functional polarization of cone horizontal cell dendrites and synapses. Science 190:989-990. CrossRef Medline

Stephens GC, Fingerman M, Brown FA (1953) The orientation of Drosophila to plane polarized light. Ann Entomol Soc Am 46:75-83.

Strausfeld NJ, Wunderer H (1985) Optic lobe projections of marginal ommatidia in Calliphora erythrocephala specialized for detecting polarized light. Cell Tissue Res 242:163-178.

Stuart AE (1999) From fruit flies to barnacles, histamine is the neurotransmitter of arthropod photoreceptors. Neuron 22:431-433. CrossRef Medline

Takemura SY, Bharioke A, Lu Z, Nern A, Vitaladevuni S, Rivlin PK, Katz WT, Olbris DJ, Plaza SM, Winston P, Zhao T, Horne JA, Fetter RD, Takemura S, Blazek K, Chang LA, Ogundeyi O, Saunders MA, Shapiro V, Sigmund C, et al. (2013) A visual motion detection circuit suggested by Drosophila connectomics. Nature 500:175-181. CrossRef Medline

Tomlinson A (2003) Patterning the peripheral retina of the fly. Dev Cell 5:799-809. Medline 
Velez MM, Wernet MF, Clark DA, Clandinin TR (2014) Walking Drosophila align with the e-vector of linearly polarized light through directed modulation of angular acceleration. J Comp Physiol A Neuroethol Sens Neural Behav Physiol 200:603-614. Medline

Wada S (1974) Special marginal ommatidia of flies (Diptera: Brachycera): Architecture and distribution in compound eyes-Spezielle randzonale Ommatidien der Fliegen (Diperta: Brachycera): Architektur und Verteilung in den Komplexaugen. Z Morphol Tiere 77:87-125.

Wehner R, Labhart $\mathrm{T}$ (2006) Polarization vision. In: Invertebrate vision (Warrant E, Nilsson D, eds), pp 291-348. Cambridge, UK: Cambridge UP.

Weir PT (2013) Polarization-based navigation in Drosophila. Doctoral dissertation, California Institute of Technology.

Weir PT, Dickinson MH (2012) Flying Drosophila orient to sky polarization. Curr Biol 22:21-27. CrossRef Medline

Wernet MF, Labhart T, Baumann F, Mazzoni EO, Pichaud F, Desplan C
(2003) Homothorax switches function of Drosophila photoreceptors from color to polarized light sensors. Cell 115:267-279. CrossRef Medline

Wernet MF, Velez MM, Clark DA, Baumann-Klausener F, Brown JR, Klovstad M, Labhart T, Clandinin TR (2012) Genetic dissection reveals two separate retinal substrates for polarization vision in Drosophila. Curr Biol 22:12-20. CrossRef Medline

Wilson RI, Turner GC, Laurent G (2004) Transformation of olfactory representations in the Drosophila antennal lobe. Science 303:366-370. CrossRef Medline

Wolf R, Gebhardt B, Gademann R, Heisenberg M (1980) Polarization sensitivity of course control in Drosophila melanogaster. J Comp Physiol 139:177-191.

Wunderer H, Smola U (1982) Fine structure of ommatidia at the dorsal eye margin of Calliphora erythrocephala Meigen (Diptera: Calliphoridae): An eye region specialised for the detection of polarized light. Inl J Insect Morphol Embryol 11:25-38. 